\title{
Predictive capacity of thigh muscle strength in symptomatic and/or radiographic knee osteoarthritis progression - data from the FNIH OA Biomarkers Consortium
}

\author{
Adam G Culvenor, PT, PhD ${ }^{1,2}$, Wolfgang Wirth, $\mathrm{PhD}^{1,3}$, Melanie Roth, Mag ${ }^{1}$, David J Hunter, \\ MBBS, PhD ${ }^{4}$, and Felix Eckstein, MD ${ }^{1,3}$ \\ ${ }^{1}$ Institute of Anatomy, Paracelsus Medical University Salzburg \& Nuremberg, Salzburg, AUSTRIA \\ ${ }^{2}$ School of Allied Health, La Trobe University, Bundoora, AUSTRALIA \\ ${ }^{3}$ Chondrometrics $\mathrm{GmbH}$, Ainring, GERMANY \\ ${ }^{4}$ Rheumatology Department, Royal North Shore Hospital and Institute of Bone and Joint \\ Research, Kolling Institute, University of Sydney, Sydney, AUSTRALIA
}

\section{Abstract}

Thigh muscle weakness is a risk factor for incident radiographic and symptomatic knee osteoarthritis. The role of thigh muscle weakness in radiographic and/or symptomatic knee osteoarthritis progression remains elusive. 527 knees of 527 Osteoarthritis Initiative participants with baseline Kellgren-Lawrence grade 1-3 were included in this nested case-control study evaluating whether baseline muscle strength predicted symptomatic and/or radiographic knee osteoarthritis progression. Case knees $(\mathrm{n}=173)$ displayed both medial tibiofemoral joint space loss $(\searrow 0.7 \mathrm{~mm}$ ) and a persistent increase in Western Ontario McMasters (WOMAC) pain ( $\ 9$ on a 0 100 scale) over 24-48 months from baseline. Control knees ( $n=354)$ included 174 with neither radiographic nor symptomatic progression, 91 with radiographic progression only, and 89 with symptomatic progression only. Isometric knee extensor and flexor strength were recorded at baseline. Using logistic regression models, muscle strength was not associated with case status. However, knee extensor (odds ratio $=1.7 ; 95 \%$ confidence interval 1.1, 3.3; $\mathrm{p}=0.035$ ) and flexor weakness (odds ratio $=2.0 ; 95 \%$ confidence interval $1.1,3.3 ; \mathrm{p}=0.016$ ) predicted isolated symptomatic progression in males, but not in females. The results indicate that thigh muscle strength may affect symptomatic and structural progression differently in males with knee osteoarthritis, and identify an important window for potentially lowering risk of symptomatic osteoarthritis progression in men.

\section{Keywords}

osteoarthritis; knee; progression; muscle strength

\footnotetext{
Address for correspondence: Dr Adam G Culvenor, Institute of Anatomy, Paracelsus Medical University, Strubergasse 21, A5020 Salzburg, AUSTRIA, Tel: +43 6622420 80417; Fax: +43 662442002 1249; adam.culvenor@ pmu.ac.at.

Potential conflict of interest:

Adam Culvenor and Melanie Roth have no conflict of interest to report.
} 


\section{INTRODUCTION}

Thigh muscle weakness has been commonly observed in individuals with knee osteoarthritis (KOA). In literature reviews, low thigh muscle strength has been identified as a risk factor for incident radiographic ${ }^{1}$, and symptomatic $\mathrm{KOA}^{2}$. The specific role of thigh muscle weakness in radiographic and/or symptomatic progression of KOA, however, remains elusive. Some studies reported knee extensor weakness to represent a risk factor of radiographic ${ }^{3}$ and symptomatic ${ }^{4} \mathrm{KOA}$ progression in females, but not in males, while other studies found no significant association of muscle strength with either radiographic or symptomatic progression ${ }^{5,6}$. Since radiographic and symptomatic KOA progression often occur in combination, these inconsistent observations may reflect a distinct or even differential relationship of thigh muscle strength with different types of progression (i.e., combined and/or isolated symptomatic and/or radiographic) that have not yet been elucidated. Thigh muscle strength may influence symptomatic and radiographic progression differently; knee symptoms and muscle strength are both mediated by the central nervous system ${ }^{7}$, whereas structural disease is not ${ }^{8}$. Greater knowledge on whether indeed thigh muscle weakness is a risk factor for these different types of progression is clinically important, as muscle strength is a potential modifiable risk factor. Identifying distinct relationships between strength and progression may therefore affect current nonpharmacological treatment approaches of KOA.

The Foundation for the National Institutes of Health (FNIH) OA Biomarkers Consortium was established to investigate the predictive validity of various disease progression biomarkers $^{9}$ in the Osteoarthritis Initiative (OAI). Having identified OAI participants with combined and isolated radiographic or symptomatic KOA progression ${ }^{9}$, the FNIH consortium provides an ideal platform from which to evaluate the prognostic capacity and association of change of thigh muscle weakness on different types of progression ${ }^{10}$. The specific aims of the current study were to determine: i) whether baseline thigh muscle weakness is a risk factor for combined or isolated radiographic and/or symptomatic progression; and ii) whether there exists a significant association of longitudinal change in thigh muscle strength with concurrent radiographic and/or symptomatic progression vs. no KOA progression.

\section{MATERIAL AND METHODS}

\section{Participants}

This study was ancillary to the FNIH OA Biomarkers Consortium project, consisting of a nested case-control study using OAI data ${ }^{9}, 10,11$. The OAI is a multi-center longitudinal cohort study with 4,796 participants who were examined annually over four years using imaging, clinical and biospecimen (i.e., urine/serum) outcomes ${ }^{12}$. To be eligible for the FNIH OA Biomarkers cohort, participants needed to have $\geq 1$ knee with baseline tibiofemoral Kellgren-Lawrence grade (KLG) 1-3, and availability of 24 month (M) radiographs, clinical data, biospecimens, and magnetic resonance images (MRI) 9 . Participants with knee or hip arthroplasty up to $24 \mathrm{M}$ were excluded. A flow diagram detailing FNIH project participant recruitment has been recently published elsewhere ${ }^{11}$. For the current analysis, participants also needed to have knee extensor and flexor strength data 
at baseline and 24M, which were available for 527 of the $600 \mathrm{FNIH}$ consortium participants (Table 1). The study was approved by the local Institutional Review Boards at each of the OAI sites, and participants provided informed consent.

Severity of KOA (KLG) was assessed by central reading of fixed-flexion radiographs and semi-quantitative joint space narrowing (JSN), while pain was assessed using the Western Ontario McMasters (WOMAC) pain subscale. Minimum radiographic joint space width (minJSW) in the medial tibiofemoral compartment was assessed using automated software $^{12}$, with established cross-sectional (ICC 0.98) and longitudinal (ICC 0.96) reliability (http://www.oai.ucsf.edi/datarelease/). Knees with lateral compartment JSN grades 2-3 at baseline or poorly positioned radiographs were excluded, as this may limit the accuracy of medial tibiofemoral minJSW measurements, or result in radiographic misclassification $^{13}$.

Radiographic progression was defined as medial compartment minJSW loss of $\searrow 0.7 \mathrm{~mm}$ from baseline to 24,36 or $48 \mathrm{M}$. The $0.7 \mathrm{~mm}$ threshold was based on the $12 \mathrm{M}$ change distribution in minJSW in OAI control participants; it involves a minimal probability ( $\triangleleft 0 \%$ ) of change due to measurement error ${ }^{9}, 14$. Symptomatic progression was defined as a persistent increase of $\geq 9$ points on a $0-100$ normalized WOMAC score from baseline to 24 , 36,48 or $60 \mathrm{M}$, i.e. a minimally clinically important difference ${ }^{15}$. Persistent pain cases were those who reported increased pain $\geq 9$ WOMAC points vs. baseline at $\geq 2$ time points from $24-60 \mathrm{M}^{9}$. Knees that reached radiographic and symptomatic progression status between baseline and 12M were excluded. The specific outcome groups are detailed in Table 1.

\section{Procedures}

The maximal isometric extensor and flexor forces (Newtons $[\mathrm{N}]$ ) were determined as the maximum of three attempts at $60^{\circ}$ knee flexion (Good Strength Chair; Metitur Oy). Baseline and 24M data reported in the OAI database were used (clinical data BL/Y2: 0.2.2/3.2.1) ${ }^{16}$. Four female participants had flexor strength $<10 \mathrm{~N}$ (three at baseline, one at $24 \mathrm{M}$ ), whereas extensor strength was within a normal range $(78-248 \mathrm{~N})$ in all participants. Because the measurement of flexor strength was likely inaccurate, these four observations were excluded from current analyses. Muscle strength data was normalized to body weight $(\mathrm{N} / \mathrm{kg})$ for analysis. Because OAI muscle strength measurements were taken at an anatomically consistent location, the isometric force measurements $(\mathrm{N} / \mathrm{kg})$ were used directly, and not moments, consistent with previous OAI strength evaluations ${ }^{16}$. The rationale for this was that the lever arm between the load cell and joint center, and that between the muscle tendons and joint center, both depend on body size; they may thus be assumed to be roughly proportional ${ }^{16}$, so that the measured force provides an accurate representation of the actual muscle force. Although the lever arm between the load cell and joint center was documented for some OAI participants, these measurements were only available in 281 (53\%) of the 527 participants.

Demographic, structural and pain characteristics of the primary case (Group 1) and control groups (Group 1,2 and 3) were compared using parametric and non-parametric tests as appropriate (based on normality) and chi-square tests for data in contingency tables. The ability of baseline muscle strength to predict progression status was evaluated using logistic 
regression, after confirming normality. The association of change in muscle strength from baseline to $24 \mathrm{M}$ with concurrent KOA progression status was also evaluated using logistic regression, with both analyses performed separately in male and female participants. Odds ratios (OR) and 95\% confidence intervals (CI) per standard deviation were reported for each muscle strength outcome after adjusting for age, body mass index (BMI), baseline KLG, and pain frequency status in the past year (none, infrequent, frequent). An OR $>1$ represents greater odds of KOA progression occurring in the presence of muscle weakness or in the presence of greater strength loss over 24M. In the primary, pre-specified analysis of FNIH data, muscle strength outcomes were compared between primary case knees (Group 1) and control knees (Groups 2-4). Secondary analyses compared muscle strength outcomes between combined and isolated radiographic/symptomatic progression groups (Group 1, 2 and 3) vs. the no progression group (Group 4), respectively. All analyses were performed with SPSS, V22.0. P-values $<0.05$ were considered significant.

\section{RESULTS}

Of the 194 cases and 406 controls included in the primary FNIH Biomarker cohort, 173 (89\%) cases and 354 (87\%) controls had muscle strength assessment at both baseline and 24M (Table 1). There were no statistically or clinically relevant differences in age, BMI, baseline KLG or pain frequency between those who did and did not have muscle strength recorded $(\mathrm{p}<0.05)$. Baseline characteristics of participants included in the current study appear in Table 2, with the full FNIH cohort baseline data having been published previously ${ }^{10,11}$. The baseline demographics of the primary cases (Group 1) and controls (Group 2, 3 and 4) were similar (Table 2). The primary cases had more severe OA (KLG and medial JSN) and more pain medication use.

The mean $(95 \% \mathrm{CI})$ muscle strength for each of the primary and secondary KOA status groups at baseline and 24M are shown in Figure 1. In the primary analysis, baseline muscle strength did not predict combined radiographic and symptomatic progression compared to controls without this combination (Table 3). Male and female primary cases (group 1) and controls (groups 2-4) all lost knee extensor (range of means: -0.26 to $-0.40 \mathrm{~N} / \mathrm{kg}$ ) and flexor strength (range: -0.15 to $-0.24 \mathrm{~N} / \mathrm{kg}$ ) from baseline to $24 \mathrm{M}$ ( $\mathrm{p}<0.003$ ). Strength loss over $24 \mathrm{M}$ was not associated with combined radiographic and symptomatic progression case status in male or female participants when compared to those without combined progression (Table 3).

In secondary comparisons, both baseline knee extensor (adjusted OR [aOR] 1.7, 95\% CI 1.1, 3.3) and flexor weakness (aOR 2.0, 95\% CI 1.1,3.3) in male subjects predicted isolated symptomatic progression (Group 3 ) compared to male subjects without symptomatic or radiographic progression (Group 4). Female participants with isolated symptomatic progression also had lower baseline extensor strength than all other groups, but the difference did not reach statistical significance in comparison with controls without symptomatic or radiographic progression (aOR 1.4, 95\% CI 0.9, 2.0). Baseline flexor strength of female subjects with isolated symptomatic progression was similar to subjects free of symptomatic and radiographic progression (aOR 1.3, 95\% CI 0.9, 2.0). Male participants with symptomatic progression started with low baseline extensor strength and 
lost less knee extensor strength over 24M than those in all other groups (Figure 1), but this difference did not reach statistical significance in comparison with those without symptomatic or radiographic progression (aOR $0.8,95 \% \mathrm{CI} 0.5,1.3)$. Similar non-significant observations were observed in knee extensor strength loss in females with isolated symptomatic progression compared to those free of symptomatic and radiographic progression (aOR $0.8,95 \% \mathrm{CI} 0.6,1.1)$. Less loss of knee flexor strength in males between baseline and 24M was associated with JSN progression compared to those free of symptomatic and radiographic progression (aOR $0.6,95 \% \mathrm{CI} 0.4,0.9)$, due to the relatively large strength loss in the controls (Group 4 ) (Figure 1). No such associations between longitudinal change in muscle strength and progression were observed in female participants (Table 3).

\section{DISCUSSION}

In this first evaluation of thigh muscle strength as a predictor of distinct combinations of symptomatic and radiographic progression, results show that the relationship between knee extensor and flexor weakness and KOA progression differs depending on the type of progression evaluated, particularly for males. For example, while baseline and $24 \mathrm{M}$ change in thigh muscle strength in male participants did not predict symptomatic progression in the presence of radiographic progression, both knee extensor and flexor weakness predicted isolated symptomatic progression in the absence of radiographic progression. Because joint effusion, pain, trauma and decreased activity all lead to muscle weakness in a vicious cycle, these results may also apply to earlier phases of KOA. These results identify a window for potentially lowering risk of symptomatic KOA progression in men.

The findings that knee extensor and flexor strength predicted symptomatic progression in male subjects, but only when simultaneous radiographic progression was actively ruled out, suggest that different mechanisms may drive pain in males with KOA depending on the type of progression, and thigh muscle strength may affect symptomatic and structural progression differently. The results in males extend recent observations using OAI data that demonstrated thigh muscle strength to be more strongly associated with symptomatic KOA than radiographic $\mathrm{KOA}^{16}$. Based on previous relationships between muscle strength and KOA progression reported in females ${ }^{3,4}$, it was surprising that no relationship was observed between muscle strength and any type of KOA progression in female knees. However, differences in methodology may explain these different results. For example, previous studies have included females without, but at risk of, KOA, and therefore evaluated incident symptoms or structural deterioration rather than progression per $\mathrm{se}^{3,4}$. It is conceivable that females at risk of KOA may have different relationships with clinical outcomes from those with established $\mathrm{KOA}^{17}$. Furthermore, knee replacement was included in previous progression definitions ${ }^{3,4}$ but not in the current study. The decision to undergo knee replacement is in part driven by factors independent of structural or symptomatic status. Importantly, the results may help explain why few associations between thigh muscle strength and OA progression have been previously observed in males, when different types of progression (i.e., symptomatic vs. radiographic) were analyzed together without being clearly dissected from each other ${ }^{3,4}$. Based on the results of this study, isolated symptomatic 
progression and symptomatic progression in combination with radiographic progression should not be considered as one 'symptomatic entity', especially in men.

Concurrent change in thigh muscle strength was not associated with the primary outcome of combined symptomatic and radiographic progression. Yet, male participants who lost less knee flexor strength over 24M exhibited isolated radiographic progression. The perhaps unexpected relationship between less loss of strength and KOA progression in this group may have been due to the low knee flexor strength at baseline, resulting in non-significant loss of strength over $24 \mathrm{M}$, and a larger loss of strength in those with neither radiographic nor symptomatic progression. In females, in contrast, all of the progression groups displayed very similar strength loss trajectories over $24 \mathrm{M}$.

This study evaluated whether knee extensor or flexor weakness predicts KOA progression because both groups of thigh muscles contribute to knee joint stability. Weakness in either the knee extensors or flexors could reduce neuromuscular control and shock absorption, which may be associated with knee symptoms. On the other hand, given that joint loads are largely determined by muscle force, greater muscle strength may be associated with greater joint loads, and hence be related to greater structural (radiographic) progression due to mechanical challenges, which may not immediately translate to knee symptoms. For these reasons, the relationship between muscle strength and the different types of progression may be complex and difficult to elucidate.

A limitation of the current study is that not all participants eligible for the FNIH OA biomarker cohort had muscle strength data recorded at baseline and 24M. However, $>85 \%$ of primary cases and controls were included, and these participants did not differ in terms of age, sex, BMI, baseline pain frequency or radiographic KOA status from those who did not have muscle strength measurement. Subgrouping males and females separately in all four subgroups implied some risk of potential chance findings, given a number of parallel exploratory analyses that were not adjusted for multiple comparisons. However, it was important to analyze males and females separately because of the greater variability in the observed muscle strength when mixing males and females, and because previous studies reported that males and females display different muscle strength risk profiles for KOA progression ${ }^{3,4}$. The main finding, that baseline muscle weakness in male participants predicted symptomatic progression, was consistent in both knee extensors and flexors, suggesting a genuine relationship between muscle weakness and symptomatic progression in males, and only two sub-groups had $<50$ participants, with narrow CIs indicating relatively precise estimates of effect sizes (Table 3). Some of the findings may also be influenced by the presence of patellofemoral OA, which was not assessed in the current study because skyline radiographs, used to assess patellofemoral pathology, were not part of the OAI data collection protocol.

\section{CONCLUSION}

In conclusion, baseline knee extensor and flexor muscle weakness predicted KOA symptomatic progression in males, but not in females. This association was lost when radiographic and symptomatic progression occurred together. These results indicate that 
different mechanisms may drive pain in men with KOA, depending on the type of progression, and that thigh muscle strength may affect symptomatic and structural progression differently. Given the modifiable nature of muscle strength deficits, these results may identify a new window to lower the risk of symptomatic KOA progression in men.

\title{
Supplementary Material
}

Refer to Web version on PubMed Central for supplementary material.

\section{Acknowledgments}

\begin{abstract}
Funding:
Scientific and financial support for the FNIH OA Biomarkers Consortium have been made possible through grants, direct and in-kind contributions provided by: AbbVie; Amgen Inc.; Arthritis Foundation; Bioiberica S.A.; DePuy Mitek, Inc.; Flexion Therapeutics, Inc.; GlaxoSmithKline; Merck Serono; Rottapharm | Madaus; Sanofi; Stryker; The Pivotal OAI MRI Analyses (POMA) Study, and the NIH HHSN2682010000. The OAI is a public-private partnership comprised of five contracts (N01-AR-2-2258; N01-AR-2-2259; N01-AR-2-2260; N01-AR-2-2261; N01-AR-2-2262) funded by the National Institutes of Health. Funding partners include Merck Research Laboratories; Novartis Pharmaceuticals Corporation, GlaxoSmithKline; and Pfizer, Inc. Private sector funding for the Consortium and OAI is managed by the FNIH. This research has received funding from the European Union Seventh Framework Programme (FP7-PEOPLE-2013-ITN; KNEEMO) under grant agreement number 607510. The sponsors were not involved in the design and conduct of this particular study, in the analysis and interpretation of the data, and in the preparation, review, or approval of the manuscript.

Wolfgang Wirth has a part time employment with Chondrometrics GmbH and is a co-owner of Chondrometrics $\mathrm{GmbH}$, a company providing MRI analysis services to academic researchers and to industry. Felix Eckstein is CEO of Chondrometrics $\mathrm{GmbH}$; he has provided consulting services to Merck Serono, Mariel Therapeutics, Servier, and Bioclinica/Synarc, has prepared educational sessions for Medtronic, and has received research support from Pfizer, Eli Lilly, Merck Serono, GlaxoSmithKline, Centocor R\&D, Wyeth, Novartis, Stryker, Abbvie, Kolon, Synarc, Ampio, BICL and Orthotrophix. David Hunter has received support from the FNIH OA Biomarkers Consortium.
\end{abstract}

\section{REFERENCES}

1. Oiestad BE, Juhl CB, Eitzen I, Thorlund JB. Knee extensor muscle weakness is a risk factor for development of knee osteoarthritis. A systematic review and meta-analysis. Osteoarthritis Cartilage. 2015; 23:171-177. [PubMed: 25450853]

2. Segal NA, Glass NA. Is quadriceps muscle weakness a risk factor for incident or progressive knee osteoarthirtis. Phys Sportsmed. 2011; 39:44-50. [PubMed: 22293767]

3. Segal NA, Glass NA, Torner J, Yang M, Felson DT, Sharma L, et al. Quadriceps weakness predicts risk for knee joint space narrowing in women in the MOST cohort. Osteoarthritis Cartilage. 2010; 18:769-775. [PubMed: 20188686]

4. Glass NA, Torner JC, Frey Law LA, Wang K, Yang T, Nevitt MC, et al. The relationship between quadriceps muscle weakness and worsening of knee pain in the MOST cohort: a 5-year longitudinal study. Osteoarthritis Cartilage. 2013; 21:1154-1159. [PubMed: 23973125]

5. Brandt KD, Heilman DK, Slemenda C, Katz BP, Mazzuca SA, Braunstein EM, et al. Quadriceps strength in women with radiographically progressive osteoarthritis of the knee and those with stable radiographic changes. J Rheumatol. 1999; 26:2431-2437. [PubMed: 10555906]

6. Amin S, Baker K, Niu J, Clancy M, Goggins J, Guermazi A, et al. Quadriceps strength and the risk of cartilage loss and symptom progression in knee osteoarthritis. Arthritis Rheum. 2009; 60:189198. [PubMed: 19116936]

7. Alnahdi AH, Zeni JA, Snyder-Mackler L. Muscle impairements in patients with knee osteoarthritis. Sport Health. 2012; 4:284-292.

8. Bastick AN, Belo JN, Runhaar J, Bierma-Zeinstra SMA. >What are the prognostic factors for radiographic progression of knee osteoarthritis? A meta-analysis. Clin Orthop Relat Res. 2015; 473:2969-2989. [PubMed: 25995176] 
9. Hunter DJ, Nevitt M, Losina E, Kraus V. Biomarkers for osteoarthritis: current position and steps towards further validation. Best Pract Res Cl Rh. 2014; 28:61-71.

10. Eckstein F, Collins JE, Nevitt MC, Lynch JA, Kraus V, Katz JN, et al. Cartilage thickness change as an imaging biomarker of knee osteoarthritis progression: Data from the Foundation for the National Institutes of Health Osteoarthritis Biomarkers Consurtium. Arthritis Rheumatol. 2015; 67:3184-3189. [PubMed: 26316262]

11. Hunter DJ, Nevitt M, Lynch J, Kraus VB, Katz JN, Collins JE, et al. Longitudinal validation of periarticular bone area and 3D shape as biomarkers for knee OA progression? Data from the FNIH OA Biomarkers Consortium. Ann Rheum Dis. 2015 (Epub ahead of print).

12. Eckstein F, Wirth W, Nevitt M. Recent advances in osteoarthrits imaging - the Osteoarthritis Initiative. Nat Rev Rheumatol. 2012; 8:622-630. [PubMed: 22782003]

13. Wirth W, Nevitt M, Hellio Le Graverand MP, Lynch J, Maschek S, Hudelmaier M, et al. Lateral and medial joint space narrowing predict subsequent cartilage loss in the narrowed, but not in the non-narrowed femorotibial compartment: data from the Osteoarthritis Initiative. Osteoarthritis Cartilage. 2014; 22:63-70. [PubMed: 24185111]

14. Ornetti P, Brandt KD, Hellio Le Graverand MP, Hochberg M, Hunter DJ, Kloppenburg M, et al. OARSI-OMERACT definition of relevant radiological progression in hip/knee osteoarthritis. Osteoarthritis Cartilage. 2009; 17:856-863. [PubMed: 19230857]

15. Angst F, Aeschlimann A, Stucki G. Smallest detectable and minimal clinically important differences of rehabilitation intervention with their implications for required sample sizes using WOMAC and SF-36 quality of life measurement instruments in patients with osteoarthrits of the lower extremities. Arthritis Rheumatol. 2001; 45:384-391.

16. Ruhdorfer A, Wirth W, Hitzl W, Nevitt M, Eckstein F. Association of thigh muscle strength with knee symptoms and radiographic disease stage of osteoarthritis: data from the osteoarthritis initiative. Arthrit Care Res. 2014; 66:1344-1353.

17. Schiphof D, Van Middelkoop M, de Klerk BM, Oei EHG, Hofman A, Koes BW, et al. Crepitus is a first indication of patellofemoral osteoarthritis (and not tibiofemoral osteoarthritis). Osteoarthritis Cartilage. 2014; 22:631-638. [PubMed: 24583066] 

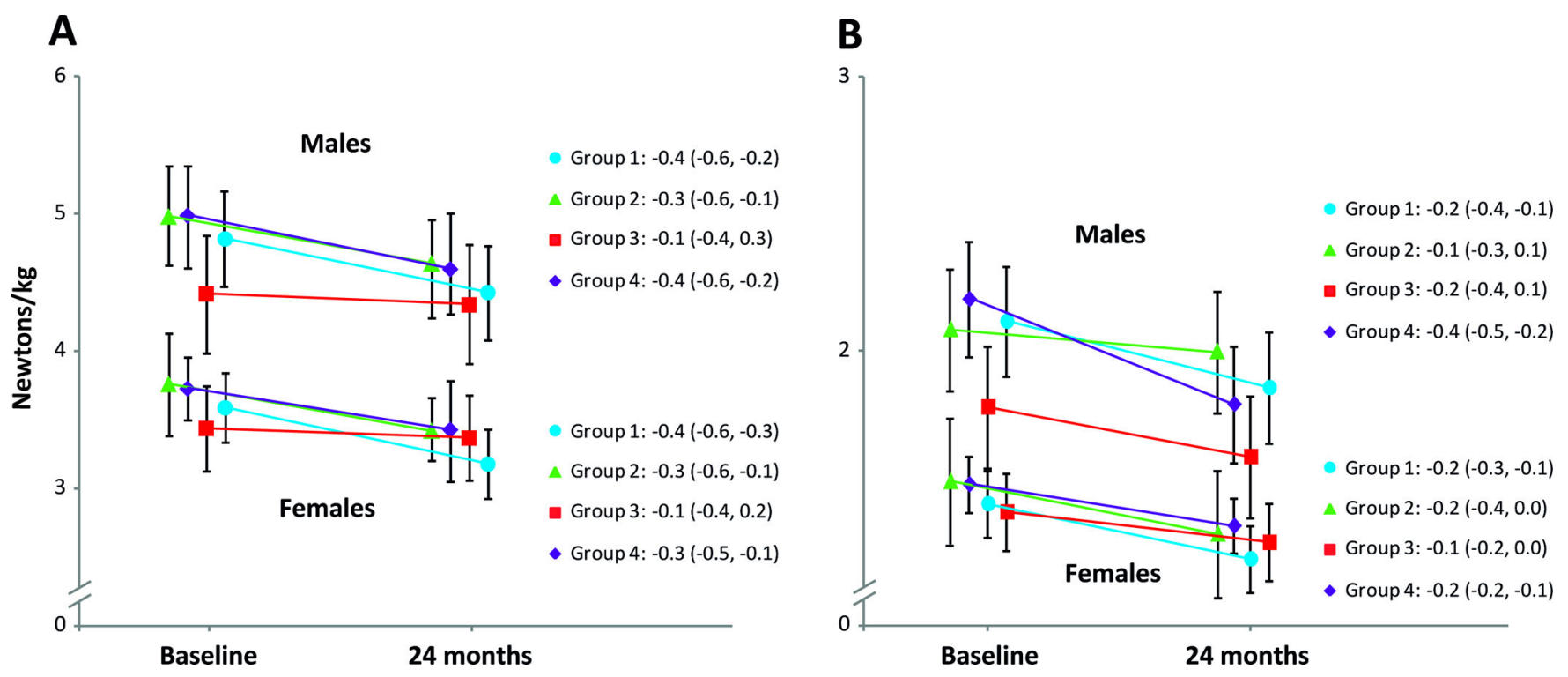

Figure 1.

Knee extensor (A) and flexor (B) muscle strength (Newtons $/ \mathrm{kg}$ ) and 95\% confidence intervals for males and females at baseline and 24 months. Values written in text for each group represent mean change in muscle strength (95\% confidence interval) from baseline to 24 months.

Group 1: knees with both radiographic and pain progression; Group 2: knees with isolated radiographic progression; Group 3: knees with isolated pain progression; Group 4: knees with neither radiographic nor pain progression. 


\section{Table 1}

Outcome groups for the FNIH OA Biomarker cohort.

\begin{tabular}{lcc}
\hline Group Description $\S$ & $\begin{array}{c}\text { Participants in } \\
\text { full dataset }\end{array}$ & $\begin{array}{c}\text { Participants in } \\
\text { current study }\end{array}$ \\
\hline$\underline{\text { Primary Cases }}$ & 194 & 173 \\
1. Knees with both radiographic and pain progression & 103 & 91 \\
Controls \# & 103 & 89 \\
2. Knees with radiographic but not pain progression & 200 & 174 \\
3. Knees with pain but not radiographic progression & & \\
4. Knees with neither radiographic nor pain progression & & \\
\hline
\end{tabular}

${ }^{\#}$ Controls were made up of participants who met all eligibility criteria to be cases except the concurrent radiographic and symptomatic progression criteria.

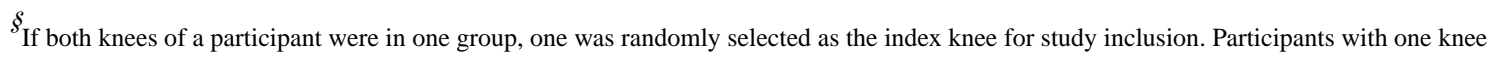
displaying only radiographic progression and the other knee only pain progression were excluded. Knees selected for the four groups were frequency matched to the extent possible using KLG strata $1-3$ and body mass index strata $<25,25-27.5,27.5-30,30-35,235 \mathrm{~kg} / \mathrm{m}^{2}$. Group 4 knees could not have radiographic progression, including worsening of lateral compartment JSN, or pain progression in the contralateral knee.

F, females; M, males 


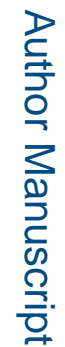

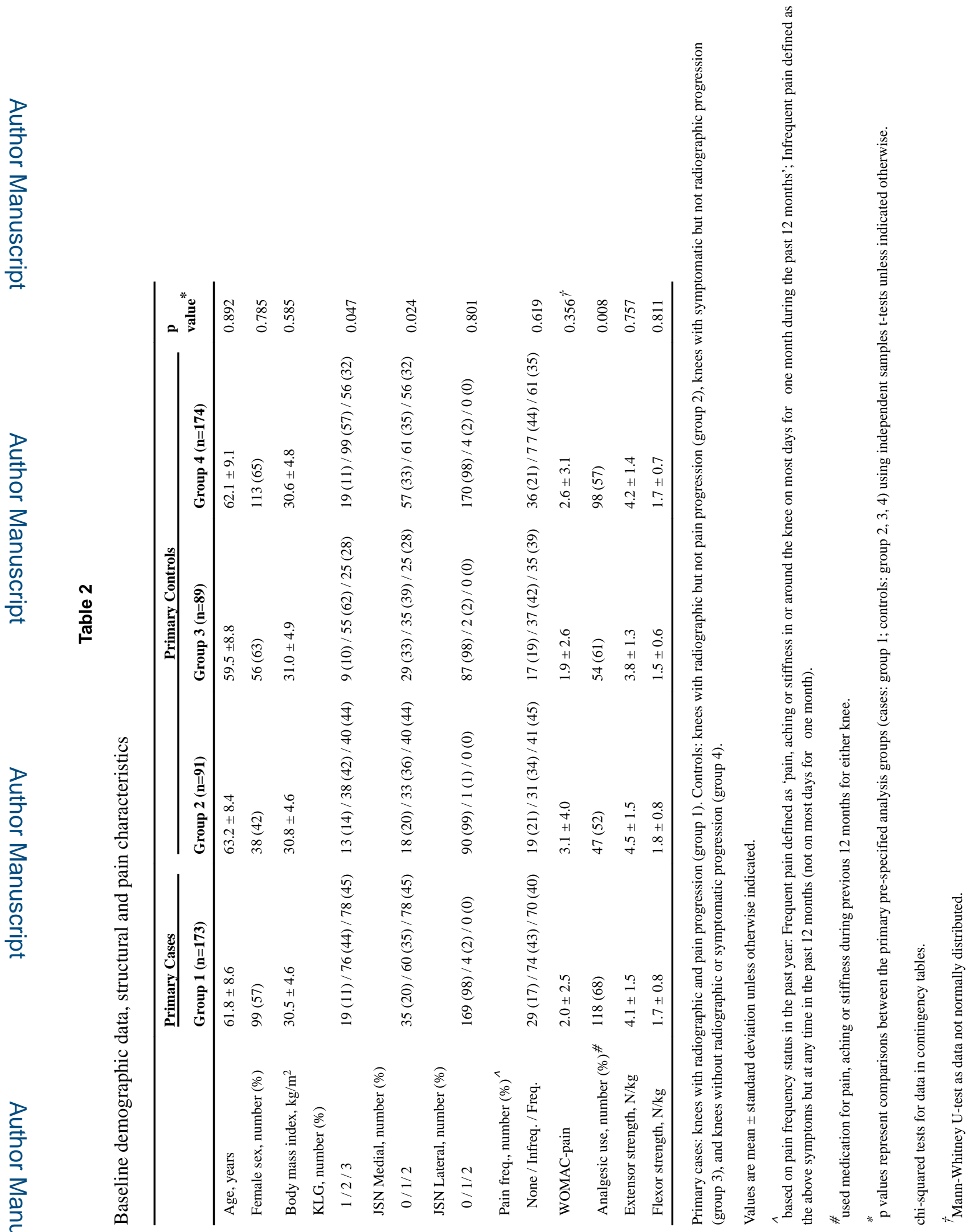

Am J Phys Med Rehabil. Author manuscript; available in PMC 2017 December 01. 


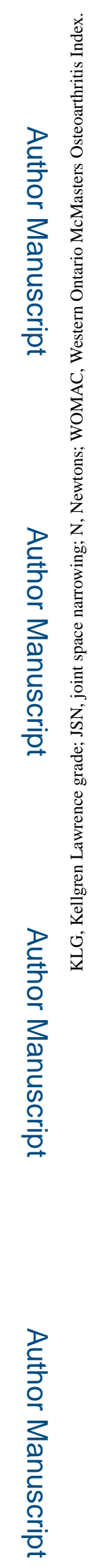




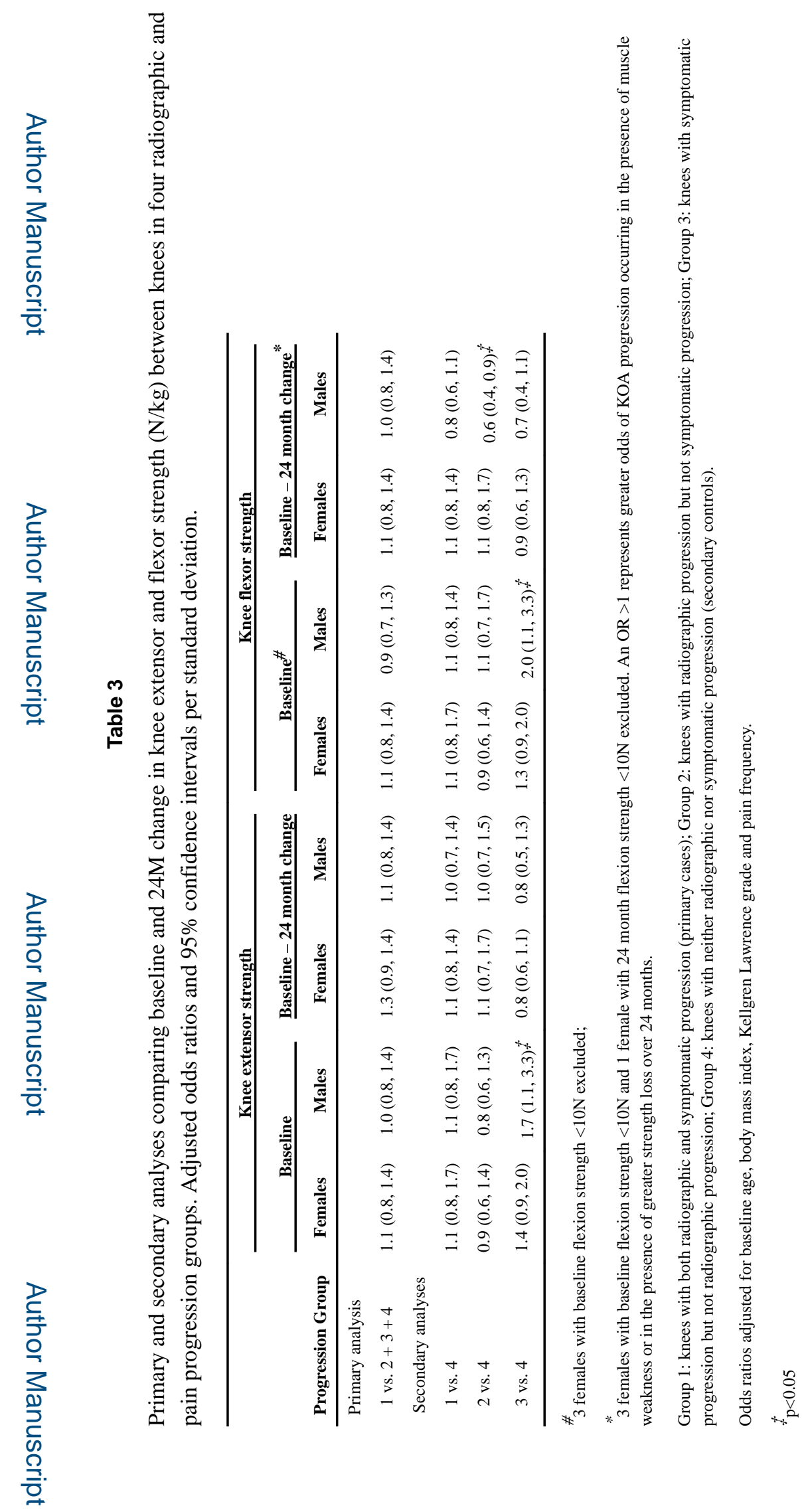

Am J Phys Med Rehabil. Author manuscript; available in PMC 2017 December 01. 